\title{
Minimum reporting guidelines and the role of causal inference in functional neuroimaging for obesity research
}

\author{
Tyler Davis ${ }^{1} \cdot$ Hans-Rudolf Berthoud ${ }^{2} \cdot$ Martin Binks $^{3}$
}

Received: 24 March 2020 / Revised: 8 April 2020 / Accepted: 14 May 2020 / Published online: 28 May 2020

(c) Springer Nature Limited 2020

Neuroimaging methods are commonplace in behavioral and clinical research, including nutritional science. Given the wide range of questions technologies like functional magnetic resonance imaging (fMRI) have been used to test, there is considerable variation in the methodologies used. With such high methodological variability in the field, there is increasing consensus of a need for minimum standards for fMRI data collection, analysis, and reporting. Accordingly, guidelines for minimum standards, such as the COBIDAS report guidelines released by the Organization for Human Brain Mapping [1] have arisen, and researchers have begun to shape these into more field specific roadmaps for future research. For example, recently, Smeets et al. [2] offered a set of minimum guidelines for nutritional research that echoes many of the key COBIDAS guidelines, while highlighting additional field specific issues. Their recommendations to adhere to COBIDAS, increase sample sizes, and promote greater use of longitudinal and interventionbased study designs will undoubtedly lead to improvements in the quality of publications in nutritional sciences.

Beyond following these minimum guidelines and recommendations, it is also critical to carefully consider how current methodological practices and analysis approaches in nutritional fMRI research relate to the most meaningful, field-specific research goals and objectives. Since a primary focus of many researchers in the International Journal of Obesity readership is in understanding the causal mechanisms underlying ingestive behavior and obesity, it is

Tyler Davis

tyler.h.davis@ttu.edu

1 Department of Psychological Sciences, Texas Tech University, Lubbock, TX 79403, USA

2 Department of Neurobiology \& Nutrition, Louisiana State University, Baton Rouge, LA 70808, USA

3 Department of Nutritional Sciences, Texas Tech University, Lubbock, TX 79403, USA useful to consider how the goal of causal inference relates to methodological choices one can make in fMRI research. Here we discuss model specification and research design choices in that context.

Effective statistical model specification is one of the primary challenges for causal inference from fMRI data. FMRI data are considerably larger than many data sources in nutritional research. Statistical algorithms must be applied to capture relevant variation in the data and then distill such variation to a format that makes inferences tractable. A typical example of this approach is to apply linear regression models individually to every voxel (3D pixel) in the brain and then make inferences about the spatial distribution of voxels with significant regression coefficients for variables of interest. However, because the number of voxels tested is substantial, this approach creates a notoriously large false positive problem, correcting for which creates nearly as large a problem with statistical power. A timeless solution to this problem, when considering causal inference, is to manage the size of the hypothesis space a priori by defining regions of interest (ROIs) instead of applying models to every voxel in the brain. A priori ROIs can then be examined using standard causal modeling approaches, or more brain specific biophysical models [3] with a much more manageable impact on the false positive rate. In the field of ingestive behavior, there is well-developed brain research in both humans and animals to specify such a priori models (for reviews, see $[4,5])$.

Despite the importance of ROI-based research for development of causal theories in neuroimaging and psychological brain research, there is a growing trend away from such standard causal frameworks (e.g., [6]). Predictive modeling approaches, driven by the growing popularity of "big data" machine learning techniques are becoming popular in many areas of clinical neuroimaging. Although machine learning techniques offer efficient solutions for predicting outcomes from large quantities of neuroimaging data, results of many such algorithms tend to be less easily 
interpretable than traditional, regression-based causal modeling. First, it can be difficult to understand what aspects of a signal are driving the predictions. Second, in order to cope with large amounts of data, many popular techniques require either variable selection or regularization to avoid overfitting. These techniques are necessary to maintain the efficiency of statistical estimators in large data spaces, but come with the drawback of adding bias to the model that limits causal interpretability. For example, regularization techniques can eliminate or reduce the effects of noisier variables, which can have the effect of favoring variables more proximally related to an outcome over more distal primary causes. For example, if the goal of an analysis is to predict food desirability, variable selection techniques may favor motor cortex, the most proximal region to a behavioral response, while missing more critical reward and valuation circuits. Thus the brain regions identified as predictive of ingestion-related outcomes using highly regularized, machine learning techniques have no guarantee of being the most causally relevant to interventions or theory. Instead, the results of such analyses often give only an exploratory set of brain regions that then must be further examined via more standard, ROI-based causal modeling.

A second, related model specification issue is the under specification of the causal relationships between variables of interest and control (or confound) variables that are included in statistical models. As is common in correlational research, clinical neuroimaging studies tend to use multiple regression analyses to statistically suppress the influence control variables may have on neuroimaging results. However, including such variables in models without an understanding of how they relate causally to outcome variables and variables of interest in the study leads, in many cases, to uninterpretable results, and can even wrongly falsify important hypotheses and new leads for the field [7]. For example, whether "controlling for" variables like hunger leads to useful results depends on how we think hunger contributes to brain region activation and the clinical outcome variable of interest, and is not indicated in all cases [8]. Take, for example, a clinical trial investigating a treatment that affects satiety hormone levels, which impact hunger and brain response to food images. Automatically controlling for hunger in such a scenario may mask the effect of both treatment and satiety hormones (and weaken or mask the effect of hunger itself) on the brain response. Likewise, for the vast majority of variables that impact both clinical outcomes and brain function, it is not possible to specify a causal order between brain function and the variables at all, making the utility of automatic control for such variables highly suspect. In the absence of a well-specified causal model of how potential control variables relate to other "person-related" individual difference variables and outcomes, it may be more desirable to simply establish and replicate empirical, zero-order correlations amongst variables of interest prior to moving toward more complex structural models.

In order to move forward with building more accurate causal models of ingestive behavior, more work will be needed both in model specification and study design. In terms of specifying neural circuitry, animal research has played a critical role in inspiring many human neuroimaging studies on obesity and ingestive behavior, such as links between the dopaminergic reward system and obesity [9]. Establishing that such circuitry is preserved amongst humans and animals is a critical first step, but to understand human causes of obesity, it is important to move toward a better understanding of how human cognition modifies such circuits. Indeed, cognitive control capacities likely play a large role in the failure of dietary interventions, but such capacities go beyond what is possible to test in animal models. Further, in order to understand the causal mechanisms underlying such failures, it is important to continually move toward more longitudinal and interventional designs, like those used with animal models [4]. Such designs can help to clarify the causal role of many of the brain regions found to activate differently between participants with obesity and controls.

While interventional and longitudinal designs can help with understanding how macro-level brain function contributes to obesity and ingestive behavior, to have a better understanding of the specific computations underlying regional activation, advances in behavioral imaging paradigms are also needed. Frequently used paradigms like food cue reactivity and resting state are excellent for predicting individual differences in brain function, but given the lack of precision in the constructs they measure, they are less able to test more fine-grained hypotheses about the algorithms underlying the observed neural circuits. For example, finding that participants with and without obesity differ in resting state connectivity in networks associated with executive control does not necessarily answer how such control processes may actually differ, because resting state does not measure control per se. Indeed, it is not possible to infer, based on such differences, that any specific cognitive functions differ between participants as brain networks do not correspond to cognitive states in a one-to-one manner [10]. Improving task designs to better measure such constructs, along with incorporation of mathematical models that provide quantitative causal hypotheses for how such processes are engaged temporally in a task, will be critical for improving our understanding of the causal mechanisms underlying the brain regions that contribute to ingestive behavior.

In conclusion, neuroimaging research has great promise for the field of obesity and ingestive behavior. Developing minimum standards for study design and reporting are critical for neuroimaging to reach its full potential. 
Beyond this minimum standard, researchers need to be aware of how different model specification choices and study designs limit the potential for strong causal inference from neuroimaging results.

\section{Compliance with ethical standards}

Conflict of interest The authors declare that they have no conflict of interest.

Publisher's note Springer Nature remains neutral with regard to jurisdictional claims in published maps and institutional affiliations.

\section{References}

1. Nichols TE, Das S, Eickhoff SB, Evans AC, Glatard T, Hanke M, et al. Best practices in data analysis and sharing in neuroimaging using MRI. Nat Neurosci. 2017;20:299-303.

2. Smeets PA, Dagher A, Hare TA, Kullmann S, van der Laan LN, Poldrack RA, et al. Good practice in food-related neuroimaging. Am J Clin Nutr. 2019;109:491-503.
3. Havlicek M, Roebroeck A, Friston K, Gardumi A, Ivanov D, Uludag K. Physiologically informed dynamic causal modeling of fMRI data. Neuroimage. 2015;122:355-72.

4. Kahathuduwa CN, Boyd LA, Davis T, O'Boyle M, Binks M. Brain regions involved in ingestive behavior and related psychological constructs in people undergoing calorie restriction. Appetite. 2016;107:348-61.

5. Berthoud HR, Münzberg H, Morrison CD. Blaming the brain for obesity: integration of hedonic and homeostatic mechanisms. Gastroenterology. 2017;152:1728-38.

6. Yarkoni T, Westfall J. Choosing prediction over explanation in psychology: lessons from machine learning. Perspect Psychol Sci. 2017;12:1100-22.

7. Meehl PE. High school yearbooks: a reply to Schwarz. J Abnorm Psychol. 1971;77:143-8.

8. Chin SH, Kahathuduwa CN, Stearns MB, Davis T, Binks M. Is hunger important to model in fMRI visual food-cue reactivity paradigms in adults with obesity and how should this be done? Appetite. 2018;120:388-97.

9. Volkow ND, Wang GJ, Baler RD. Reward, dopamine and the control of food intake: implications for obesity. Trends Cogn Sci. 2011;15:37-46.

10. Poldrack RA. Can cognitive processes be inferred from neuroimaging data? Trends Cogn Sci. 2006;10:59-63. 